\title{
BAER AND MITTAG-LEFFLER MODULES OVER TAME HEREDITARY ALGEBRAS
}

\author{
LIDIA ANGELERI HÜGEL, DOLORS HERBERA, AND JAN TRLIFAJ
}

\begin{abstract}
We develop a structure theory for two classes of infinite dimensional modules over tame hereditary algebras: the Baer modules, and the Mittag-Leffler ones. A right $R$-module $M$ is called Baer if $\operatorname{Ext}_{R}^{1}(M, T)=0$ for all torsion modules $T$, and $M$ is Mittag-Leffler in case the canonical map $M \otimes_{R} \prod_{i \in I} Q_{i} \rightarrow \prod_{i \in I}\left(M \otimes_{R} Q_{i}\right)$ is injective where $\left\{Q_{i}\right\}_{i \in I}$ are arbitrary left $R$-modules.

We show that a module $M$ is Baer iff $M$ is $\mathbf{p}$-filtered where $\mathbf{p}$ is the preprojective component of the tame hereditary algebra $R$. We apply this to prove that the universal localization of a Baer module is projective in case we localize with respect to a complete tube. Using infinite dimensional tilting theory we then obtain a structure result showing that Baer modules are more complex then the (infinite dimensional) preprojective modules. In the final section, we give a complete classification of the Mittag-Leffler modules.
\end{abstract}

Since the fundamental work of Ringel [29], the study of infinite dimensional modules has become one of the challenging tasks of the representation theory of finite dimensional hereditary algebras. In the present paper, we consider in detail two classes of infinite dimensional modules over tame hereditary algebras: the Baer modules, and the Mittag-Leffler ones.

Besides proving structure results, we investigate further the surprising analogy with modules over Dedekind domains discovered in [29. Indeed, the current progress relies heavily on applications of the recent powerful set-theoretic and homological methods developed originally for modules over domains, cf. [3] and [15].

Baer modules can be defined in a rather general setting 26: Let $R$ be a ring and $\mathcal{T}$ be a torsion class in Mod- $R$. A module $M \in \operatorname{Mod}-R$ is a Baer module for $\mathcal{T}$ provided that $\operatorname{Ext}_{R}^{1}(M, T)=0$ for all $T \in \mathcal{T}$.

In the particular case when $R=\mathbb{Z}$, and more generally when $R$ is a integral domain and $\mathcal{T}$ is the class of all torsion $R$-modules, we obtain thus the classical notions of a Baer group 8 and a Baer module 17. It took quite a long time to prove that these notions actually coincide with the well-known notions of a free group and a projective module, respectively. Countable Baer groups were shown to be free already in 1936 by Baer [8], but the arbitrary ones only in 1969 by Griffith [16]. The projectivity of the classical Baer modules was also shown in two steps spreading over decades, however, in a different order. First, a reduction to countably presented modules was proved by set-theoretic methods by Eklof, Fuchs

\footnotetext{
Date: October 29, 2018.

Part of this research was done during the visit of the authors to CRM Barcelona in September 2006 supported by the Research Programme on Discrete and Continuous Methods of Ring Theory. First author partially supported by Università di Padova, Progetto di Ateneo CDPA048343, and by PRIN 2005 "Prospettive in teoria degli anelli, algebre di Hopf e categorie di moduli". First two authors partially supported by the DGI and the European Regional Development Fund, jointly, through Project MTM2005-00934, and by the Comissionat per Universitats i Recerca of the Generalitat de Catalunya, Project 2005SGR00206. Third author acknowledges support by GAČR 201/06/0510 and MSM 0021620839.
} 
and Shelah [12] in 1990. Finally, the countably presented case has recently been settled in [3].

In the present paper, we consider Baer modules over tame hereditary algebras, so our $\mathcal{T}$ is the class of all torsion modules in the sense of Ringel [29] (see below for unexplained terminology). Baer modules in this sense have been studied since 1980's, notably by Okoh and Lukas in 24, 26, and 22. Their focus was on countably presented modules, but their results already indicated the complexity of the general case.

Here we take a different approach. First, in Section 1, we apply set-theoretic methods to prove a reduction to finitely presented modules. We infer that the Baer modules are precisely the modules filtered by the finitely generated preprojective modules (Theorem 21). This reveals the main difference from the integral domain case: the role of the finitely generated projectives is taken by the much more complex class of the finitely generated preprojectives.

However, as shown in Section 2, the reduction is still sufficient to imply that Baer modules are locally projective, in the sense that their universal localizations, as introduced by Schofield in [31, are projective in case we localize with respect to a set of simple regular modules containing a complete clique (Theorem 5).

In Section 3 we employ infinite dimensional tilting theory in order to investigate the structure of the infinite dimensional torsion-free, and Baer modules. The key role is played here by an infinite dimensional tilting module, the so called Lukas tilting module $L$. It is a module which has no finite dimensional direct summands, not even pure-injective direct summands, but it is noetherian when viewed as a module over its endomorphism ring (Corollary 9).

Baer modules are characterized as the kernels of homomorphisms between modules in $\operatorname{Add} L$, and torsion-free modules as the pure-epimorphic images of Baer modules with kernels in $\operatorname{Add} L$ (Corollaries 10 and 11).

We recover Lukas' theorem on the structure of countably presented Baer modules [22, and extend it to arbitrary Baer modules (Corollary 15); then we establish a bijective correspondence between equivalence classes of Baer modules and isomorphism classes of (infinite dimensional) preprojective modules (Corollary 18).

Also the Mittag-Leffler modules can be defined in a very general setting [30, [5]: Given a class of left $R$-modules $\mathcal{Q}$, we call a module $M \mathcal{Q}$-Mittag-Leffler if the canonical map $M \otimes_{R} \prod_{i \in I} Q_{i} \rightarrow \prod_{i \in I}\left(M \otimes_{R} Q_{i}\right)$ is injective for any family $\left\{Q_{i}\right\}_{i \in I}$ of left $R$-modules in $\mathcal{Q}$. For $\mathcal{Q}=R$-Mod, this is just the well-known notion of a Mittag-Leffler module from [27].

If $R$ is a tame hereditary algebra then the connection with Baer modules is that every Baer module $M$ is $\mathcal{C}$-Mittag-Leffler where $\mathcal{C}$ denotes the class of all left $R$-modules without non-zero preinjective direct summands.

For artin algebras, it is known that Mittag-Leffler modules coincide with the separable modules, that is, the modules $M$ whose finite subsets are always contained in finitely presented direct summands 35]; we observe that they also coincide with the strict Mittag-Leffler modules, that is, with locally pure-projective modules in the sense of 7 . We prove that in the setting of tame hereditary algebras, the torsion-free separable modules coincide with the pure submodules of products of indecomposable preprojective modules (Proposition 21), while the torsion reduced separable modules coincide with the locally split epimorphic images of direct sums of indecomposable finitely generated regular modules (Proposition 23).

Then we prove the main result of Section 4, a structure theorem for Mittag-Leffler modules (Theorem 27). 
All our rings are unital, and by the unadorned term $(R$-)module we mean a right module over the ring $R$.

We will use the following notation. For a class of modules $\mathcal{C}$, we define

and

$$
\begin{aligned}
& { }^{o} \mathcal{C}=\left\{M \in \operatorname{Mod}-R \mid \operatorname{Hom}_{R}(M, C)=0 \text { for all } C \in \mathcal{C}\right\}, \\
& { }^{{ }} \mathcal{C}=\left\{M \in \operatorname{Mod}-R \mid \operatorname{Ext}_{R}^{1}(M, C)=0 \text { for all } C \in \mathcal{C}\right\}
\end{aligned}
$$

$$
{ }^{\perp} \mathcal{C}=\left\{M \in \operatorname{Mod}-R \mid \operatorname{Ext}_{R}^{i}(M, C)=0 \text { for all } C \in \mathcal{C} \text { and all } i>0\right\} .
$$

Similarly, the classes $\mathcal{C}^{o}, \mathcal{C}^{\perp_{1}}$ and $\mathcal{C}^{\perp}$ are defined.

We will need some notions related to purity. A monomorphism $\iota: N \rightarrow M$ is said to be s-pure (or strongly pure or locally split) if for any finite subset $S \subseteq N$ there is an $R$-homomorphism $\pi: M \rightarrow N$ such that $\pi \iota(x)=x$ for each $x \in S$. According to [35], a module $M$ is said to be locally pure-injective if every pure-monomorphism $M \rightarrow X$ with $X \in$ Mod- $R$ is s-pure.

An $s$-pure submodule $N$ of a module $M$ is a submodule with the property that the embedding $\iota: N \rightarrow M$ is s-pure. Replacing the term "finite subset" by "countable subset", we obtain the notion of a $c$-pure submodule of a module $M$. Clearly, we have the implications: $N$ is a direct summand in $M \Rightarrow N$ is c-pure in $M \Rightarrow N$ is s-pure in $M \Rightarrow N$ is pure in $M$.

We will also use the dual notions. A homomorphism $\pi: X \rightarrow M$ is a locally split epimorphism if for each finite subset $F \subseteq M$ there is a map $\varphi=\varphi_{F}: M \rightarrow X$ such that $m=\pi \varphi(m)$ for all $m \in F$. A module $M$ is said to be strict Mittag-Leffler (or locally pure-projective) if every pure-epimorphism $X \rightarrow M$ with $X \in \operatorname{Mod}-R$ is locally split, see [27] and [7.

Finally, recall that a module $T$ is said to be a (1-) tilting module if it satisfies

(T1) proj.dim $(T) \leq 1$;

(T2) $\operatorname{Ext}_{R}^{1}\left(T, T^{(I)}\right)=0$ for each set $I$; and

(T3) there is an exact sequence $0 \rightarrow R \rightarrow T_{0} \rightarrow T_{1} \rightarrow 0$ where $T_{0}$ and $T_{1}$ are direct summands of a (possibly infinite) direct sum of copies of $T$.

\section{BAER MODUles AND p-Filtrations}

We start with a general setting and show that set-theoretic methods allow to reduce the structure of Baer modules to the countably generated ones.

Let $\sigma$ be an ordinal. An increasing chain of submodules, $\mathcal{M}=\left(M_{\alpha} \mid \alpha \leq \sigma\right)$, of a module $M$ is called a filtration of $M$ provided that $M_{0}=0, M_{\alpha}=\bigcup_{\beta<\alpha} M_{\beta}$ for all limit ordinals $\alpha \leq \sigma$ and $M_{\sigma}=M$.

Given a class of modules $\mathcal{C}$ and a module $M$, a filtration $\mathcal{M}$ is a $\mathcal{C}$-filtration of $M$ provided that $M_{\alpha+1} / M_{\alpha}$ is isomorphic to some element of $\mathcal{C}$ for each $\alpha<\sigma$. In this case we say that $M$ is $\mathcal{C}$-filtered.

Given a cardinal $\kappa$, a filtration $\mathcal{M}$ is a $\kappa$-filtration of $M$ provided that $M_{\alpha}$ is $<\kappa$-generated for each $\alpha<\sigma$.

Theorem 1. Let $R$ be an $\aleph_{0}-$ noetherian ring and $\mathcal{T}$ be a torsion class in $\mathrm{Mod}-R$ such that ${ }^{\perp_{1}} \mathcal{T}={ }^{\perp} \mathcal{T}$. Assume that either $\mathcal{T}$ consists of modules of finite injective dimension, or ${ }^{\perp} \mathcal{T}$ consists of modules of finite projective dimension. Then the following conditions are equivalent for any module $M$ :

(1) $M$ is a Baer module for $\mathcal{T}$.

(2) $M$ has a filtration $\mathcal{M}=\left(M_{\alpha} \mid \alpha \leq \kappa\right)$ such that, for each $\alpha<\kappa, M_{\alpha+1} / M_{\alpha}$ is a countably generated Baer module for $\mathcal{T}$. 
Proof. 1. implies 2. The case when ${ }^{\perp} \mathcal{T}$ consists of modules of finite (and hence bounded) projective dimension is a particular instance of [15, Theorem 4.3.10] (for $\nu=\aleph_{0}$ and $n=$ the common bound of the projective dimensions of all modules in $\perp \mathcal{T})$.

If $\mathcal{T}$ consists of modules of finite injective dimension, then (as $\mathcal{T}$ is closed under arbitrary direct sums) there is $n<\omega$ such that $\mathcal{T} \subseteq \mathcal{I}_{n}$ where $\mathcal{I}_{n}$ denotes the class of all modules of injective dimension $\leq n$. We will proceed by a modification of the proof of [15, Theorem 10.1.5] (for $\mu=\aleph_{0}$ ). Since the latter proof has an extra set-theoretic assumption of the Weak Diamond, we will give more details below indicating how to avoid this assumption by employing the fact that $\mathcal{T}$ is closed under arbitrary direct sums.

For each $i \leq n$, let $\mathcal{A}_{i}={ }^{\perp}\left(\mathcal{T} \cup \mathcal{I}_{i}\right)$. Then $\mathcal{A}_{i}={ }^{\perp} \mathcal{T} \cap{ }^{\perp} \mathcal{I}_{i}={ }^{\perp_{1}} \mathcal{T} \cap{ }^{\perp_{1}} \mathcal{I}_{i}$. Denote by $\mathcal{Q}_{i}$ a representative set of all countably generated modules in $\mathcal{A}_{i}$. By downward induction on $i$, we will prove that each module in $\mathcal{A}_{i}$ is $\mathcal{Q}_{i}$-filtered (our claim 2. is then the case of $i=0$, since ${ }^{\perp} \mathcal{I}_{0}=\operatorname{Mod}-R$.)

For $i=n$ we have $\mathcal{A}_{i}={ }^{\perp} \mathcal{I}_{n}$. From Baer's criterion of injectivity and the fact that all syzygies of cyclic modules can be taken countably generated (since $R$ is $\aleph_{0}$-noetherian) we deduce that the cotorsion pair $\left({ }^{\perp} \mathcal{I}_{n}, \mathcal{I}_{n}\right)$ is generated by a class of $<\aleph_{1}$ - presented modules. So the claim follows from [15, Theorem 4.2.11].

The induction step from $i+1$ to $i$ for $i<n$ is proved by transfinite induction on the minimal number $\lambda$ of $R$-generators of $M \in \mathcal{A}^{i}$ as in [15, Theorem 10.1.5]. The only place where the Weak Diamond is used there is when $\lambda$ is regular and uncountable, for selecting a subfiltration with consecutive factors in $\perp \mathcal{T}$ from a $\lambda$-filtration of $M$ consisting of modules in ${ }^{\perp} \mathcal{T}$. However, this is possible by [15, Theorem 4.3.2] since $\mathcal{T}$ is closed under arbitrary direct sums.

The implication 2. implies 1. is a particular case of the well-known Eklof Lemma (see e.g. [15, Lemma 3.1.2]).

From now on, unless stated otherwise, we will assume that $R$ is an indecomposable tame hereditary artin algebra with standard duality $D: \bmod -R \rightarrow R-\bmod$ between right and left finitely generated $R$-modules, respectively.

We recall the notation of [28] and [29]: $\mathbf{t}$ and $\mathbf{p}$ will denote representative sets of all indecomposable finitely generated regular, and preprojective, modules, respectively. Further,

$$
\mathcal{F}=\mathbf{t}^{o}={ }^{\perp} \mathbf{t}
$$

is the torsion-free class of all torsion-free modules. Note that

$$
\mathcal{F} \cap \bmod -R=\text { addp, hence } \mathcal{F}=\underline{\text { lim}} \text { addp. }
$$

Since add $\mathbf{t}$ is closed under extensions, the corresponding torsion class, called the class of all torsion modules, is Gent, see [28, 3.5].

A module $M$ is a Baer module provided that $M$ is a Baer module for Gent. The class of all Baer modules is denoted by $\mathcal{B}$. So

$$
\mathcal{B}={ }^{\perp} \text { Gent } \subseteq \mathcal{F}={ }^{\perp} \mathbf{t}, \quad \text { and } \quad \mathcal{B} \cap \bmod -R=\mathcal{F} \cap \bmod -R=\operatorname{add} \mathbf{p}
$$

Remark. Baer modules over tame hereditary algebras are torsion-free, and the same holds for the classical Baer modules over commutative integral domains. However, this fails in general: Let $R$ be a ring and $T$ be a tilting module. Take $\mathcal{T}=T^{\perp}$. This is the torsion class consisting of all modules generated by $T$, and the corresponding torsion-free class is $T^{o}$. Thus $T$ is a Baer module for $\mathcal{T}$ which is not torsion-free.

The modules having no non-zero homomorphism to $\mathbf{p}$ are called $\mathcal{P}^{\infty}$-torsion, and their class is denoted by $\mathcal{L}$. By the infinitary version of the Auslander-Reiten 
formula 11

$$
\mathcal{L}={ }^{o} \mathbf{p}=\mathbf{p}^{\perp}
$$

so $\mathcal{L}$ is a tilting torsion class. There is a countably infinitely generated tilting module generating $\mathcal{L}$, called the Lukas tilting module, and denoted by $L$, cf. 18 .

For a module $M$, we will denote by $l(M)$ the $\mathcal{P}^{\infty}$-torsion submodule of $M$, that is, $l(M)$ is the trace of $L$ in $M$. The torsion-free class corresponding to $\mathcal{L}$ will be denoted by $\mathcal{P}$. This is the class of all (possibly infinitely generated) preprojective modules (Warning: in 21] and [22, preprojective modules are called ' $\mathcal{P}{ }^{\infty}$-torsionfree'). We have

$$
\mathcal{P}=L^{o}=\left({ }^{o} \mathbf{p}\right)^{o} \subseteq \mathbf{t}^{o}=\mathcal{F}, \quad \text { and } \quad \mathcal{P} \cap \bmod -R=\operatorname{add} \mathbf{p}
$$

Moreover, if $M$ is a module such that $M \notin \mathcal{L}$, then $M$ has a non-zero finitely generated preprojective direct summand (see [29, Corollary 2.2]). In particular, the preprojective indecomposable modules are precisely the copies of modules from p. Similarly, the finitely $\mathbf{p}$-filtered modules are exactly the modules in addp.

In our particular setting, thanks to a result from 26, we can improve the reduction Theorem 1 and obtain the following characterization of Baer modules:

Theorem 2. Let $R$ be a tame hereditary artin algebra and $M$ be a module. Then $M$ is a Baer module if and only if $M$ is $\mathbf{p}$-filtered.

Proof. Assume $M \in \mathcal{B}$. By Theorem 1, $M$ has a filtration $\mathcal{M}=\left(M_{\alpha} \mid \alpha \leq \kappa\right)$ such that $M_{\alpha+1} / M_{\alpha}$ is a countably generated Baer module for each $\alpha<\kappa$. So w.l.o.g., we can assume that $M$ is countably generated. Then, by 26, Theorem 1.2], $M=\bigcup_{n<\omega} M_{n}$ where $M_{n}$ is finitely generated, and $M_{n+1} / M_{n} \in \mathcal{F}$ for each $n<\omega$. Since $\mathcal{B} \cap \bmod -R=\mathcal{F} \cap \bmod -R=\operatorname{add} \mathbf{p}$, we infer that $M$ has a filtration with all consecutive factors finitely generated and preprojective, and hence $M$ is p-filtered.

For the if-part, notice that since $\mathbf{p} \subseteq \mathcal{B}$, also add $\mathbf{p} \subseteq \mathcal{B}$, that is, all finitely generated preprojective modules are Baer. So the claim follows Eklof's Lemma [15. Lemma 3.1.2].

Remark. Theorem 2 is analogous to the corresponding result for integral domains. In that case, however, one has to replace "preprojective" by "projective", so the existence of the filtration $\mathcal{M}$ already yields a direct sum decomposition, and hence projectivity, of $M$, cf. [3] and [12].

Being in a hereditary ring, a submodule of a Baer module is again Baer. However, the existence of a filtration of $M$ as in Theorem 2 implies existence of a large family of Baer submodules of $M$ such that also all consecutive factors in the family are Baer:

Corollary 3. Let $R$ be a tame hereditary algebra. Let $M$ be a Baer module with a $\mathbf{p}$-filtration $\mathcal{M}$ as in Theorem 2 . Then there exists a family $\mathcal{Y}$ of (Baer) submodules of $M$ such that

(H1) $\mathcal{M} \subseteq \mathcal{Y}$ (in particular, $0, M \in \mathcal{Y})$.

(H2) $\mathcal{Y}$ is closed under arbitrary sums and intersections.

(H3) If $N, P \in \mathcal{Y}$ and $N \subseteq P$, then $P / N$ is a Baer module.

(H4) Let $N \in \mathcal{Y}$ and $X$ be a finite subset of $M$. Then there is $P \in \mathcal{Y}$ such that $N \cup X \subseteq P$ and $P / N \in$ addp.

Proof. This follows by an application of the Hill lemma [15, Theorem 4.2.6] to the filtration $\mathcal{M}$ (for $\kappa=\aleph_{0}$ ). 


\section{UNIVERSAL LOCALIZATIONS OF BAER MODULES}

Though Theorem 2 does not yield direct sum decompositions of Baer modules, it can be used to prove that universal localizations of Baer modules, in the sense of Schofield 31, always decompose in case we localize with respect to a set of isomorphism classes of simple regular modules $\mathcal{U}$ containing at least one complete clique (that is, in case the universal localization $R_{\mathcal{U}}$ is a hereditary noetherian prime ring, see [10, §4]). In fact, in this case the localized Baer modules are projective.

Recall that a clique is an equivalence class with respect to the equivalence relation induced on the set of isomorphism classes of simple regular modules by the relation $S \sim S^{\prime}$ if $\operatorname{Ext}_{R}^{1}\left(S, S^{\prime}\right) \neq 0$. In other words, two simple regular modules $S$ and $S^{\prime}$ belong to the same clique if and only if they are in the same tube. All cliques are finite, and all but finitely many consist of exactly one simple regular module.

Let $\mathcal{U}$ be a set of isomorphism classes of simple regular modules. For every $S \in \mathcal{U}$ fix a presentation

$$
0 \rightarrow P_{S} \stackrel{f_{S}}{\rightarrow} Q_{S} \rightarrow S \rightarrow 0
$$

consisting of finitely generated projective modules. The ring $R_{\mathcal{U}}$ can be described as the universal localization of $R$ at the set of maps between projective modules $\Sigma=\left\{f_{S} \mid S \in \mathcal{U}\right\}$.

In [10, Theorem 4.2], Crawley-Boevey proved that $R_{\mathcal{U}}$ is a hereditary noetherian prime ring provided that $\mathcal{U}$ contains, at least, one complete clique. Otherwise $\mathcal{U}$ is finite, and $R_{\mathcal{U}}$ is again a tame hereditary artin algebra with the same center as $R$.

For discussing the behaviour of Baer modules under localization, we will need the following preliminary observations.

Lemma 4. Let $R$ be a tame hereditary algebra. Let $\mathcal{U}$ be a set of isomorphism classes of simple regular modules. Then:

(i) [10, 2.2, §4] The canonical map $R \rightarrow R_{\mathcal{U}}$ is an inclusion, so that there is an exact sequence

$$
0 \rightarrow R \rightarrow R_{\mathcal{U}} \rightarrow N \rightarrow 0 .
$$

(ii) $\operatorname{Tor}_{1}^{R}\left(P, R_{\mathcal{U}}\right)=0=\operatorname{Tor}_{1}^{R}(P, N)$ for any finitely generated preprojective $R$ module $P$.

(iii) $\operatorname{Tor}_{1}^{R}\left(M, R_{\mathcal{U}}\right)=0=\operatorname{Tor}_{1}^{R}(M, N)$ for any Baer $R$-module $M$.

Proof. To prove $(i i)$ we note that in the short exact sequence of left $R$-modules

$$
0 \rightarrow R \rightarrow R_{\mathcal{U}} \rightarrow N \rightarrow 0
$$

the module $N$ is a directed union of left $R$-modules that are finitely $\mathcal{V}$-filtered where $\mathcal{V}=\{\operatorname{Tr} U \mid U \in \mathcal{U}\}$ and $\operatorname{Tr} U$ denotes the transpose of $U$ (see [32, Theorem 3]).

Let $P$ be any finitely generated preprojective module. For each $U \in \mathcal{U}$ we have by [32, Lemma 4] that $\operatorname{Tor}_{1}^{R}(P, \operatorname{Tr} U) \cong \operatorname{Hom}_{R}(U, P)=0$. This proves that $\operatorname{Tor}_{1}^{R}(P, V)=0$ for each $V \in \mathcal{V}$, and hence $\operatorname{Tor}_{1}^{R}(P, N)=0$. Since also $\operatorname{Tor}_{1}^{R}(P, R)=0$, we infer that $\operatorname{Tor}_{1}^{R}\left(P, R_{\mathcal{U}}\right)=0$.

(iii) follows from $($ ii $)$, because Baer modules are torsion-free, hence direct limits of finitely generated preprojective modules, and Tor commutes with direct limits.

Theorem 5. Let $R$ be a tame hereditary algebra. Let $\mathcal{U}$ be a set of isomorphism classes of simple regular modules. Denote by $R_{\mathcal{U}}$ the universal localization of $R$ with respect to $\mathcal{U}$. Let further $M$ be a Baer $R$-module, and $M_{\mathcal{U}}=M \otimes_{R} R_{\mathcal{U}}$ its universal localization.

If $\mathcal{U}$ does not contain a complete clique, then $M_{\mathcal{U}}$ is a Baer $R_{\mathcal{U}}$-module.

If $\mathcal{U}$ contains at least one complete clique, then $M_{\mathcal{U}}$ is a projective $R_{\mathcal{U}}$-module. 
Proof. By Theorem 2, $M$ has a a filtration $\mathcal{M}=\left(M_{\alpha} \mid \alpha \leq \kappa\right)$ such that $M_{\alpha+1} / M_{\alpha}=$ $P_{\alpha}$ is an indecomposable (finitely generated) preprojective module for each $\alpha<\kappa$. Since, by Lemma 4, $\operatorname{Tor}_{1}^{R}\left(P_{\alpha}, R_{\mathcal{U}}\right)=0$ for each $\alpha<\kappa$, the $R_{\mathcal{U}}$-module $M_{\mathcal{U}}=$ $M \otimes_{R} R_{\mathcal{U}}$ is a direct limit of the continuous direct system of modules

$$
\mathcal{M}_{\mathcal{U}}=\left(M_{\alpha} \otimes_{R} R_{\mathcal{U}}, f_{\beta \alpha} \mid \alpha \leq \beta \leq \kappa\right)
$$

where $f_{\beta \alpha}=u_{\beta \alpha} \otimes_{R} R_{\mathcal{U}}$ and $u_{\beta \alpha}: M_{\alpha} \rightarrow M_{\beta}$ is the inclusion. As $\operatorname{Coker}\left(f_{\alpha+1, \alpha}\right) \cong$ $P_{\alpha} \otimes_{R} R_{\mathcal{U}}$, it remains to investigate the universal localization of the indecomposable preprojective $R$-modules.

We start with the case where $\mathcal{U}$ does not contain a complete clique. Then $R_{\mathcal{U}}$ is again a tame hereditary algebra, but with a smaller rank of the Grothendieck group, see [10, Theorem 4.2]. Proceeding by induction, we can assume that $\mathcal{U}=\{S\}$ for some simple regular module $S$ in a tube of rank at least two. Then, as shown in [14, 10.1], [33, 6.9], the preprojective component $\mathbf{p}_{\mathcal{U}}$ of $R_{\mathcal{U}}$ is

$$
\mathbf{p}_{\mathcal{U}}=\mathbf{p} \cap \operatorname{Mod}-R_{\mathcal{U}}=\left\{P \otimes_{R} R_{\mathcal{U}} \mid P \in \mathbf{p}\right\}
$$

So we conclude that $M_{\mathcal{U}}$ is filtered by finitely generated preprojective $R_{\mathcal{U}}$-modules, hence it is a Baer $R_{\mathcal{U}}$-module.

Now assume that $\mathcal{U}$ contains at least one complete clique. We prove that in this case the universal localization of any indecomposable preprojective $R$-module $P$ is a nonzero projective $R_{\mathcal{U}}$-module. This will show that $M_{\mathcal{U}}$ is a projective $R_{\mathcal{U}}$-module (see [15, Lemma 3.1.5]).

Let $P$ be an indecomposable preprojective module. By assumption, there is a tube $\Theta$ whose mouth is a subset of $\mathcal{U}$. Then the embedding of $P$ into its injective envelope $E(P)$ factorizes through a finite direct sum $U=\bigoplus_{i \leq m} U_{i}$ of elements of $\Theta$. Making a pullback of a resolution of $U$

$$
0 \rightarrow P_{1} \stackrel{f}{\rightarrow} P_{0} \rightarrow U \rightarrow 0
$$

consisting of finitely generated projective modules with the inclusion $\varepsilon: P \rightarrow U$ we obtain a commutative diagram

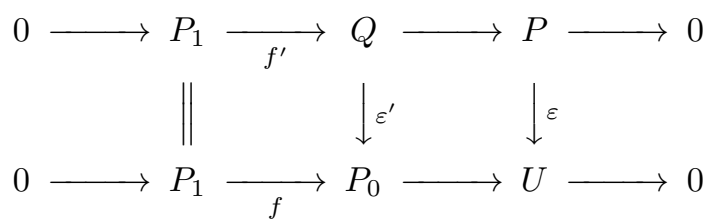

where $Q$ is projective because $\varepsilon^{\prime}$ is injective and the ring is hereditary.

As, for each $i \leq m$, all simple regular composition factors of $U_{i}$ are in $\mathcal{U}$, an inductive argument on the regular composition length of $U$ shows that $U \otimes_{R} R_{\mathcal{U}}=$ $0=\operatorname{Tor}_{R}^{1}\left(U, R_{\mathcal{U}}\right)$. Hence $f \otimes_{R} R_{\mathcal{U}}$ is bijective, and Id $=\left(f \otimes_{R} R_{\mathcal{U}}\right)^{-1}\left(\epsilon^{\prime} \otimes_{R}\right.$ $\left.R_{\mathcal{U}}\right)\left(f^{\prime} \otimes_{R} R_{\mathcal{U}}\right)$. We deduce that $P \otimes_{R} R_{\mathcal{U}}$ is a projective $R_{\mathcal{U}}$-module. Moreover as, by Lemma 4, $P$ embeds in $P \otimes_{R} R_{\mathcal{U}}$ we deduce that $P \otimes_{R} R_{\mathcal{U}}$ is a non-zero projective $R_{\mathcal{U}}$-module.

Let us now investigate the universal localization of the Lukas tilting $R$-module $L$. If $\mathcal{U}$ contains at least one complete clique then the localization is a projective $R_{\mathcal{U}}$-module by Theorem 5 . The interesting case is the one when $\mathcal{U}$ does not contain a complete clique, so $R_{\mathcal{U}}$ is again a tame hereditary algebra:

Theorem 6. Let $R$ be a tame hereditary algebra with the Lukas tilting $R$-module $L$. Let $\mathcal{U}$ be a set of isomorphism classes of simple regular modules which does not contain a complete clique. Denote by $R_{\mathcal{U}}$ the universal localization of $R$ with respect to $\mathcal{U}$. Then the universal localization $L_{\mathcal{U}}=L \otimes_{R} R_{\mathcal{U}}$ of $L$ is equivalent to the Lukas tilting $R_{\mathcal{U}}$-module $L^{\prime}$. 
Proof. As in the proof of Theorem 5 we can assume that $\mathcal{U}=\{S\}$ for a simple regular module $S$ in a tube of rank at least two. Let $\mathbf{p}_{\mathcal{U}}$ denote a representative set of all indecomposable preprojective $R_{\mathcal{U}}$-modules.

First, we claim that $\operatorname{Ext}_{R_{\mathcal{U}}}^{1}\left(P^{\prime}, L_{\mathcal{U}}\right)=0$ for each $P^{\prime} \in \mathbf{p}_{\mathcal{U}}$. By the infinitary version of the Auslander-Reiten formula, it suffices to prove that $\operatorname{Hom}_{R_{\mathcal{U}}}\left(L_{\mathcal{U}}, P^{\prime}\right)=$ 0 for each $P^{\prime} \in \mathbf{p}_{\mathcal{U}}$. Since the map $R \rightarrow R_{\mathcal{U}}$ is a ring epimorphism [31, p. 56], this is equivalent to $\operatorname{Hom}_{R}\left(L_{\mathcal{U}}, P^{\prime}\right)=0$ for each $P^{\prime} \in \mathbf{p}_{\mathcal{U}}$.

By Lemma 4, the sequence of right $R$-modules $0 \rightarrow L \rightarrow L_{\mathcal{U}} \rightarrow L \otimes_{R} N \rightarrow 0$ is exact.

We know from [14, 10.1] that $\mathbf{p}_{\mathcal{U}}=\mathbf{p} \cap \operatorname{Mod}-R_{\mathcal{U}}$. Thus $P^{\prime}$ is also preprojective as right $R$-module, so $\operatorname{Hom}_{R}\left(L, P^{\prime}\right)=0$. Similarly, $\operatorname{Hom}_{R}\left(L \otimes_{R} N, P^{\prime}\right) \cong$ $\operatorname{Hom}_{R}\left(L, \operatorname{Hom}_{R}\left(N, P^{\prime}\right)\right)=0$ since $N \cong R_{\mathcal{U}} / R$ (as a right $R$-module) is a directed union of finitely $\mathcal{U}$-filtered modules (that have no non-zero homomorphisms to $P^{\prime}$ ) by [32, Theorem 3]. Thus $\operatorname{Hom}_{R}\left(L_{\mathcal{U}}, P^{\prime}\right)=0$, and our claim is proved.

Now, we prove that $L_{\mathcal{U}}$ is a tilting module. Condition (T1) is clear since $R_{\mathcal{U}}$ is hereditary.

Condition (T2) states that $\operatorname{Ext}_{R_{\mathcal{U}}}^{1}\left(L_{\mathcal{U}}, L_{\mathcal{U}}^{(I)}\right)=0$ for any set $I$. Note that $L_{\mathcal{U}}$ is a Baer $R_{\mathcal{U}}$-module by Theorem [5, thus it is $\mathbf{p}_{\mathcal{U}}$-filtered by Theorem 2 , So, by [15, 3.1.2] it suffices to show that $\operatorname{Ext}_{R_{\mathcal{U}}}^{1}\left(P^{\prime}, L_{\mathcal{U}}^{(I)}\right)=0$ for each finitely generated preprojective $R_{\mathcal{U}}$-module $P^{\prime}$. Since $P^{\prime}$ is a finitely presented $R_{\mathcal{U}}$-module, the latter is equivalent to $\operatorname{Ext}_{R_{\mathcal{U}}}^{1}\left(P^{\prime}, L_{\mathcal{U}}\right)=0$, and (T2) follows from our claim above.

For condition (T3), consider an exact sequence $0 \rightarrow R \rightarrow L_{0} \rightarrow L_{1} \rightarrow 0$ where $L_{0}$ and $L_{1}$ are direct summands in some $L^{(J)}$. By Lemma 4 the sequence $0 \rightarrow R_{\mathcal{U}} \rightarrow$ $\left(L_{0}\right)_{\mathcal{U}} \rightarrow\left(L_{1}\right)_{\mathcal{U}} \rightarrow 0$ is exact in $\operatorname{Mod}-R_{\mathcal{U}}$, and $\left(L_{0}\right)_{\mathcal{U}},\left(L_{1}\right)_{\mathcal{U}}$ are direct summands in $L_{\mathcal{U}}^{(J)}$.

This shows that $L_{\mathcal{U}}$ is a tilting module; it is also a Baer $R_{\mathcal{U}}$-module by Theorem 5 , thus $L_{\mathcal{U}} \in{ }^{\perp}\left(L^{\prime}{ }^{\perp}\right)$. Moreover, by our claim above, $\operatorname{Ext}_{R_{\mathcal{U}}}^{1}\left(L^{\prime}, L_{\mathcal{U}}\right)=0$ because $L^{\prime}$ is $\mathbf{p}_{\mathcal{U}}$-filtered. So $L_{\mathcal{U}} \in \operatorname{Add}\left(L^{\prime}\right)$, hence $L_{\mathcal{U}}$ is equivalent to $L^{\prime}$.

Theorem [ will be used in a forthcoming paper for describing all infinite dimensional tilting modules over a tame hereditary artin algebra.

\section{The structure of Baer modules}

Throughout this section, let $R$ be a tame hereditary artin algebra. We will need further properties of the (infinitely generated) $R$-modules.

We denote by $G$ the generic module. By [29, 5.5], for each $M \in \mathcal{F}$ there are a unique cardinal $\kappa$, called the (torsion-free) rank of $M$, and an exact sequence $0 \rightarrow M \rightarrow G^{(\kappa)} \rightarrow N \rightarrow 0$ where $N$ is torsion regular.

Following 23], we call $\mathcal{E}=G^{\perp}$ the class of all cotorsion modules. In analogy with the Dedekind domain case, Okoh proved that all torsion-free cotorsion modules are pure-injective, cf. [23, p.265]. The first part of the following Proposition will give this as a consequence of (infinite dimensional) cotilting theory.

Let us first recall some basic notions. A cotorsion pair is a pair of classes of modules $(\mathcal{A}, \mathcal{B})$ such that $\mathcal{A}={ }^{\perp} \mathcal{B}$ and $\mathcal{B}=\mathcal{A}^{\perp}$. If $\mathcal{S}$ is a set of right $R$-modules, we obtain a cotorsion pair $(\mathcal{A}, \mathcal{B})$ by setting $\mathcal{B}=\mathcal{S}^{\perp}$ and $\mathcal{A}={ }^{\perp}\left(\mathcal{S}^{\perp}\right)$. It is called the cotorsion pair generated by $\mathcal{S}$. Notice that $\mathcal{A}={ }^{\perp}\left(\mathcal{S}^{\perp}\right)$ consists of all direct summands of $\mathcal{S} \cup\{R\}$-filtered modules [15, Corollary 3.2.4].

Dually, if $\mathcal{S}$ is a set of right $R$-modules, we obtain a cotorsion pair $(\mathcal{A}, \mathcal{B})$ by setting $\mathcal{A}={ }^{\perp} \mathcal{S}$ and $\mathcal{B}=\left({ }^{\perp} \mathcal{S}\right)^{\perp}$. It is called the cotorsion pair cogenerated by

\footnotetext{
${ }^{1}$ Our terminology follows [15, hence it differs from previous use.
} 
$\mathcal{S}$. Cotorsion pairs $(\mathcal{A}, \mathcal{B})($ co)generated by some (co)tilting module $M$ are called (co)tilting cotorsion pairs.

We will denote by $(\mathcal{C}, \mathcal{D})$ the cotorsion pair in $R$-Mod studied in 28 . It is generated by a representative set $\mathbf{t}^{\prime}$ of all indecomposable regular left $R$-modules, that is, $\mathcal{D}=\left(\mathbf{t}^{\prime}\right)^{\perp}$ is the class of all divisible left $R$-modules. Moreover, $(\mathcal{C}, \mathcal{D})$ is cogenerated by a representative set $\mathbf{q}^{\prime}$ of all indecomposable preinjective left $R$-modules, that is, $\mathcal{C}={ }^{\perp}\left(\mathbf{q}^{\prime}\right)$ is the class of all left modules without non-zero preinjective summands. Finally, $(\mathcal{C}, \mathcal{D})$ is a tilting and cotilting cotorsion pair (co)generated by the (co)tilting module $W$ which we call the Ringel tilting left $R$ module. Note that $W$ is the direct sum of the generic left $R$-module $G^{\prime}$ with a representative set of the Prüfer left $R$-modules. Thus $D(W)$ is the direct product of $G$ with a representative set of the adic right $R$-modules.

Proposition 7. Let $R$ be a tame hereditary algebra.

$(1)(\mathcal{F}, \mathcal{E})$ is the cotorsion pair generated by $G$. This is also the cotilting cotorsion pair cogenerated by $D(W)$. In particular, $\mathcal{F} \cap \mathcal{E}=\operatorname{Prod} D(W)$, so all torsion-free cotorsion modules are pure-injective.

$(2)(\mathcal{B}, \mathcal{L})$ is the tilting cotorsion pair generated by the tilting module $L$. It is also generated by the set $\mathbf{p}$. In particular, $\mathcal{B} \cap \mathcal{L}=\operatorname{Add} L$, and $L$ has no non-zero finitely generated direct summands.

Proof. 1. We show that ${ }^{\perp}\left(G^{\perp}\right)=\mathcal{F}$. Since $G \in \mathcal{F}$, we have ${ }^{\perp}\left(G^{\perp}\right) \subseteq \mathcal{F}$. On the other hand, if $M \in \mathcal{F}$, then $M \hookrightarrow G^{(\kappa)}$ where $\kappa$ is the torsion-free rank of $M$. Since $G \in{ }^{\perp}\left(G^{\perp}\right)$, also $M \in{ }^{\perp}\left(G^{\perp}\right)$. Moreover, $\mathbf{t}=\left\{D(X) \mid X \in \mathbf{t}^{\prime}\right\}$, thus $\mathcal{F}=\operatorname{Ker}_{\operatorname{Tor}_{1}^{R}}\left(-, \mathbf{t}^{\prime}\right)$. So, we deduce from [6, 2.3] that $\mathcal{F}$ is the cotilting torsion-free class cogenerated by the cotilting module $D(W)$. This proves the first two claims. That $\mathcal{F} \cap \mathcal{E}=\operatorname{Prod} D(W)$ follows from well-known properties of cotilting cotorsion pairs (see e.g. [15, §8.1]). As dual modules (and also cotilting modules) are pure injective, we deduce that torsion-free cotorsion modules are pure-injective.

2. By Theorem 2 and [15, 3.2.4] we have $\mathcal{B}={ }^{\perp}\left(\mathbf{p}^{\perp}\right)={ }^{\perp}\left(L^{\perp}\right)$, and the first two claims follow. That $\mathcal{B} \cap \mathcal{L}=\operatorname{Add}(L)$ follows from well-known properties of tilting cotorsion pairs (see e.g. [15, §5.1]). Finally, if $F \in \operatorname{Add} L$ is finitely generated, then $F \in \mathcal{B} \cap \bmod -R=\operatorname{add}(\mathbf{p}) \subseteq \mathcal{P}$, so $F \in \mathcal{P} \cap \mathcal{L}=\mathcal{P} \cap{ }^{o} \mathbf{p}=0$.

Example 8. We observed on page 4 that $\mathcal{B} \subseteq \lim$ addp $=\mathcal{F}$, and the finitely generated modules in both classes coincide (with the finitely generated preprojective modules). However, the two classes are different - that is, $G \in \mathcal{F} \backslash \mathcal{B}$ - because the class of all cotorsion modules is not closed under arbitrary direct sums by [23] (or because there exist preprojective modules which are not Baer, see below). In particular, $\mathcal{B}$ is not closed under direct limits.

Note furthermore that also the adic modules belong to $\mathcal{F} \backslash \mathcal{B}$. In fact, let $S_{\lambda}, \lambda \in$ $\mathbb{P}$, be a representative set of simple regular modules, and let $S_{\lambda}[-\infty], \lambda \in \mathbb{P}$, be the corresponding adic modules. If we assume that $S_{\lambda}[-\infty]$ belongs to ${ }^{\perp}\left(\mathbf{p}^{\perp}\right)$, then we obtain from [22, 3.3(a)] that every non-zero torsion-free module $Y \in \mathbf{p}^{\perp}$ satisfies $\operatorname{Hom}\left(S_{\lambda}[-\infty], Y\right) \neq 0$. But this contradicts the fact that $\operatorname{Hom}\left(S_{\lambda}[-\infty], S_{\nu}[-\infty]\right)=$ 0 whenever $S_{\lambda}, S_{\nu}$ are not in the same clique.

Remark. Notice the analogies of Proposition 7 with the case when $R$ is an integral domain:

1. In general, there are three different cotorsion pairs of $R$-modules: $(\mathcal{T} \mathcal{F}, \mathcal{R E})$ where $\mathcal{T} \mathcal{F}$ is the cotilting class of all torsion-free modules, $\left(\mathcal{F}_{0}, \mathcal{E} \mathcal{E}\right)$ where $\mathcal{F}_{0}$ is the class of all flat modules, and $(\mathcal{S F}, \mathcal{M E})$, the cotorsion pair generated by the quotient field $Q$ of $R$. Always $\mathcal{S F} \subseteq \mathcal{F}_{0}$ (the equality holds iff all proper factors of $R$ are perfect rings, [9]) and $\mathcal{F}_{0} \subseteq \mathcal{T} \mathcal{F}$ (the equality holds iff $R$ is a Prüfer domain, 
13). The cotilting module $C$ generating $\mathcal{T F}$ can be taken of the form $\delta^{*}$ where $\delta$ is the Fuchs divisible (tilting) module (see e.g. [15, Example 5.1.2]). The three cotorsion pairs coincide iff $R$ is a Dedekind domain; in this case, $C$ can be taken of the form $T^{*}=\operatorname{Hom}_{R}(T, Q / R)$ where $T=Q \oplus Q / R$ is the divisible tilting module analogous to the Ringel tilting module. (In the tame hereditary case, the analogs of the first and third cotorsion pairs coincide by Proposition 71 , but the second is the trivial cotorsion pair $\left.\left(\mathcal{P}_{0}, \operatorname{Mod}-R\right)\right)$.

2. All Baer $R$-modules are projective by [3], so the analogue of the tilting cotorsion pair $(\mathcal{B}, \mathcal{L})$ from Lemma $7(2)$ is the trivial tilting cotorsion pair $\left(\mathcal{P}_{0}, \operatorname{Mod}-R\right)$, and the analogue of the Lukas tilting module is $R$. This is reflected in the behaviour of $(\mathcal{B}, \mathcal{L})$ under universal localization at tubes, see Theorem 5 .

We now employ Proposition 7 to investigate the structure of the torsion-free $R$-modules. Let us start with the Lukas tilting module.

From Example 8 and the classification of the indecomposable pure-injective $R$ modules we conclude that $L$ has no pure-injective direct summand. In particular, $L$ is not locally pure-injective, see [35, 2.1(5)]. On the other hand, $L$ satisfies the following finiteness condition.

Corollary 9. $L$ is noetherian over its endomorphism ring.

Proof. Since $L$ is torsion-free without preprojective summands, $D(L)$ is divisible without preinjective summands, thus $D(L) \in \mathcal{C} \cap \mathcal{D}=\operatorname{Add} W$, cf. [5, 9.4]. This shows that $D(L)$ is $\Sigma$-pure-injective since so is $W$ by [28, 10.1]. Now we apply [5, 9.9] which states that a tilting module is noetherian over its endomorphism ring if and only if its dual module is $\Sigma$-pure-injective.

Corollary 10. Let $R$ be a tame hereditary algebra. A module $M$ is a Baer module if and only if there is an exact sequence $0 \rightarrow M \rightarrow L_{1} \rightarrow L_{2} \rightarrow 0$ where $L_{1}, L_{2} \in$ $\operatorname{Add} L$

Proof. This follows from Proposition 77.(2) and from the well-known characterization of modules forming tilting cotorsion pairs (see e.g. [15, Proposition 5.1.9]).

Corollary 11. The following statements are equivalent for a module $M$.

(1) $M$ is torsion-free.

(2) $M$ is a pure-epimorphic image of direct sum of indecomposable preprojective modules.

(3) $M$ occurs as the end term in a pure-exact sequence

$$
0 \rightarrow N \rightarrow B \rightarrow M \rightarrow 0
$$

with $B \in \mathcal{B}$ and $N \in \operatorname{Add} L$.

Proof. By Proposition 7 (1), each $M \in \mathcal{F}$ is a pure-epimorphic image of a direct sum of modules in addp, hence (1) implies (2).

For $(2) \Rightarrow(3)$, recall that by [15, 3.2.1] there is a special $\mathcal{B}$-precover of $M$, that is, an exact sequence $0 \rightarrow N \rightarrow B \rightarrow M \rightarrow 0$ where $B \in \mathcal{B}$ and $N \in \mathcal{L}$. Since the pure-epimorphism from (2) factors through it, the sequence is pure. Moreover, $N \in \mathcal{L} \cap \mathcal{B}=\operatorname{Add} L$ because $\mathcal{B}$ is closed under submodules.

The implication $(3) \Rightarrow(1)$ follows from the fact that $\mathcal{B} \subseteq \mathcal{F}$ and $\mathcal{F}=\underline{\lim }$ addp is closed under pure-epimorphic images.

Next, we look at the relation between $\mathcal{B}$ and $\mathcal{P}$. Since $\mathcal{P} \cap \bmod -R=\operatorname{add} \mathbf{p}$, we have $\mathcal{B} \cap \bmod -R=\mathcal{P} \cap \bmod -R$. Moreover, all preprojective modules of countable rank are Baer [22, 4.3(4)]: 
Lemma 12. Let $M$ be a preprojective module of countable rank. Then $M$ is a countably generated Baer module.

Proof. First, let $F$ be a submodule of $M$ of finite rank. We prove that $F$ is finitely generated. Indeed, if $F \neq 0$ then $F \notin \mathcal{L}$, so $F=P_{1} \oplus F_{1}$ where $0 \neq P_{1} \in$ addp. Similarly, if $F_{1} \neq 0$ then $F_{1}=P_{2} \oplus F_{2}$ for $0 \neq P_{2} \in$ addp etc. Since $F$ has finite rank, there is $n<\omega$ such that $F_{n}=0$, so $F=\bigoplus_{i \leq n} P_{i} \in$ addp is finitely generated.

So w.l.o.g., we can assume that $M$ has countably infinite rank. Consider an exact sequence $0 \rightarrow M \rightarrow G^{(\omega)}$. Then $M_{n}=M \cap G^{(n)}(n<\omega)$ are submodules of $M$ of finite rank, hence finitely generated, with the property that $M=\bigcup_{n<\omega} M_{n}$, and the factors $M_{n+1} / M_{n} \subseteq G$ belong to $\mathcal{F} \cap \bmod -R=$ addp. So $M$ is countably generated, and $M \in \mathcal{B}$ by Theorem 2 .

On the other hand, clearly, the countably generated tilting module $L$ is Baer, but not preprojective. In fact, $L$ is the only obstacle for the classes of countable rank Baer and preprojective modules to coincide. This comes from the following version of a result by Lukas ([22, Theorem 4.3(c)]):

Proposition 13. Let $M$ be a module of countable rank. Then $M$ is a Baer module if and only if $M=N \oplus P$ where $N \in \operatorname{Add} L$ and $P$ is countably generated preprojective.

Proof. The 'if' part is clear from Lemma 12, For the 'only if' part, let $M$ be a Baer module and consider the exact sequence $\varepsilon: 0 \rightarrow l(M) \rightarrow M \rightarrow M / l(M) \rightarrow 0$. Then $l(M) \in \mathcal{B} \cap \mathcal{L}=\operatorname{Add} L$. Moreover, the module $M / l(M) \in \mathcal{P}$ is of countable rank by [26, Lemma B], hence countably generated Baer by Lemma 12. Since $l(M) \in \mathcal{L}$, Lemma 7. (2) shows that $\varepsilon$ splits.

A weaker statement holds for arbitrary Baer modules. In general, the $\mathcal{P}^{\infty}$-torsion submodule $l(M)$ of a Baer module $M$ is not a direct summand, but we are going to see that is always a c-pure submodule. Actually, we prove a slightly stronger result:

Theorem 14. Let $M$ be a Baer module. Then each countable subset $C$ of $l(M)$ is contained in a countably generated submodule $D \subseteq l(M)$ which is a direct summand in $M$. In particular, $l(M)$ is a c-pure submodule in $M$.

Proof. By Proposition 13, we can w.l.o.g. assume that $M$ is not countably generated. Let $\mathcal{M}$ be an addp-filtration of $M$ coming from Theorem 2 , Let $\mathcal{Y}$ be a family of Baer submodules of $M$ corresponding to $\mathcal{M}$ by Corollary 3 . Let $\mathcal{Y}_{c}$ denote the set of all countably generated modules from $\mathcal{Y}$. By Proposition 13 for each $Y \in \mathcal{Y}_{c}$, the exact sequence

$$
0 \rightarrow l(Y) \stackrel{\nu_{Y}}{\rightarrow} Y \rightarrow Y / l(Y) \rightarrow 0
$$

splits where $\nu_{Y}$ is the inclusion of $l(Y)$ into $Y$.

We claim that $l(Y)$ is even a direct summand in $M$. Indeed, let $\rho_{Y}: Y \rightarrow l(Y)$ be such that $\rho_{Y} \nu_{Y}=\operatorname{id}_{l(Y)}$. By conditions (H1) and (H3), the module $M / Y$ is Baer, so we have the exact sequence

$$
\operatorname{Hom}_{R}(M, l(Y)) \stackrel{\operatorname{Hom}_{R}\left(\mu_{Y}, l(Y)\right)}{\longrightarrow} \operatorname{Hom}_{R}(Y, l(Y)) \rightarrow \operatorname{Ext}_{R}^{1}(M / Y, l(Y))=0
$$

where $\mu_{Y}$ is the inclusion of $Y$ into $M$. So $\rho_{Y}$ extends to some $\pi_{Y} \in \operatorname{Hom}_{R}(M, l(Y))$, and $\pi_{Y} \mu_{Y} \nu_{Y}=\operatorname{id}_{l(Y)}$ proving that $l(Y)$ is a direct summand in $M$.

Now, if $C$ is a countable subset of $l(M)$ then there exist countably many maps $f_{i} \in \operatorname{Hom}_{R}(L, M)(i<\omega)$ such that $C \subseteq S=\sum_{i<\omega} \operatorname{Im}\left(f_{i}\right)$. Since $L$ is countably generated, by conditions (H2) and (H4), there exists $Y \in \mathcal{Y}_{c}$ such that $S \subseteq Y$, and hence $C \subseteq S \subseteq l(Y)$. By the first part of the proof, $D=l(Y)$ is a direct summand in $M$. 
Remark. Notice the following peculiar property of Baer modules: each Baer module $M$ possess a $\mathbf{p}$-filtration $\mathcal{M}=\left(M_{\alpha} \mid \alpha \leq \kappa\right)$ as in Theorem 2, and in fact $M$ has many other $\mathbf{p}$-filtrations by Corollary 3 . In particular, $M$ is the well-ordered directed union of the $M_{\alpha}$ 's $(\alpha<\kappa)$. The exact sequences $0 \rightarrow l\left(M_{\alpha}\right) \rightarrow M_{\alpha} \rightarrow$ $M_{\alpha} / l\left(M_{\alpha}\right) \rightarrow 0(\alpha \leq \kappa)$ also form a well-ordered direct system. But unlike its middle term, $\mathcal{M}$, this system is not continuous in general, that is, a sequence indexed by a limit ordinal $\alpha$ need not be the direct limit of the sequences indexed by all $\beta<\alpha$. This is the price payed for $M / l(M)$ being preprojective, since direct limits of preprojective modules need not be preprojective.

This phenomenon is best seen for the Lukas tilting module $L$ : it is a directed union of a countable chain $\left(P_{n} \mid n<\omega\right)$ where all $P_{n}$ 's are finitely generated preprojective, so $l\left(P_{n}\right)=0$ for each $n<\omega$, but $l(L)=L$.

Theorem 14 yields the following generalization of Proposition 13

Corollary 15. Let $M$ be a Baer module. Then there exists a unique pure-exact sequence

$$
\varepsilon: 0 \rightarrow N \hookrightarrow M \rightarrow P \rightarrow 0
$$

such that $N=l(M) \in \operatorname{Add} L$ and $P$ is preprojective. If $l(M)$ is countably generated, then $\varepsilon$ is split exact.

Proof. By Theorem 14, the sequence $0 \rightarrow l(M) \rightarrow M \rightarrow M / l(M) \rightarrow 0$ is pureexact, and it is even split exact when $l(M)$ is countably generated. Clearly $l(M) \in$ $\mathcal{B} \cap \mathcal{L}=\operatorname{Add} L$. Uniqueness follows from the fact that $(\mathcal{L}, \mathcal{P})$ is a torsion pair in Mod-R.

Corollary 11] shows that any preprojective module occurs as the $\mathcal{P}^{\infty}$-torsion-free part of some Baer module. Such presentation is non-trivial in the uncountable rank case, since there are preprojective modules which are not Baer:

Example 16. Let $Q$ be the direct product of a set of representatives of the isoclasses from $\mathbf{p}$. Then $Q$ is preprojective (since $\mathcal{P}$ is closed under arbitrary direct products), but not Baer (see [26, Proposition 3.1]). So, $\mathcal{B}$ is not closed under direct products.

Moreover, considering the exact sequence $0 \rightarrow N \rightarrow B \rightarrow Q \rightarrow 0$ corresponding to $Q$ by Corollary 11, we see that $B$ is an uncountably generated Baer module whose $\mathcal{P}^{\infty}$-torsion submodule is pure in $B$, but does not split. So Proposition 13 cannot be extended to uncountably generated Baer modules.

Corollary[15] suggests the following definition: two Baer modules $B_{1}$ and $B_{2}$ are equivalent ( $B_{1} \sim B_{2}$ for short) provided that $B_{1} / l\left(B_{1}\right) \cong B_{2} / l\left(B_{2}\right)$.

Lemma 17. Let $R$ be a tame hereditary artin algebra and $B_{1}, B_{2} \in \mathcal{B}$. Then $B_{1} \sim$ $B_{2}$ if and only if there exist modules $L_{1}, L_{2} \in \operatorname{Add} L$ such that $B_{1} \oplus L_{1} \cong B_{2} \oplus L_{2}$.

Proof. Assume $B_{1} \sim B_{2}$, so $P=B_{1} / l\left(B_{1}\right) \cong B_{2} / l\left(B_{2}\right) \in \mathcal{P}$. Considering the pull-back of the epimorphisms $B_{1} \rightarrow P$ and $B_{2} \rightarrow P$, we obtain the following commutative diagram 


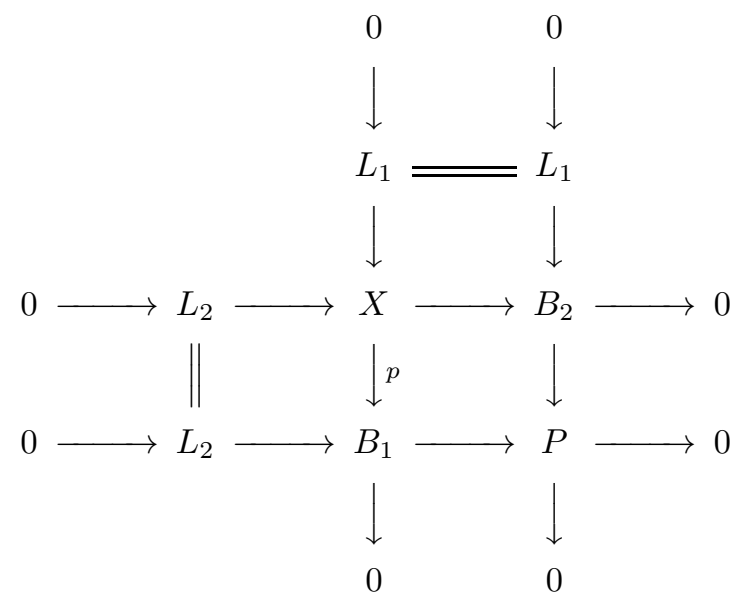

where $L_{2}=l\left(B_{1}\right)$ and $L_{1}=l\left(B_{2}\right)$ belong to $\operatorname{Add} L$. By Proposition 7 .(2), the middle row and the middle column split, so $X \cong B_{1} \oplus L_{1} \cong B_{2} \oplus L_{2}$.

The reverse implication follows by factoring out the $\mathcal{P}^{\infty}$-torsion submodule of $B_{1} \oplus L_{1} \cong B_{2} \oplus L_{2}$.

Corollary 18. There is a bijective correspondence between equivalence classes of Baer modules and isomorphism classes of preprojective modules.

Proof. The correspondence is defined on representatives as follows: $B \mapsto B / l(B)$ for a Baer module $B$; given a preprojective module $P$, we first consider a special $\mathcal{B}$-precover $B \rightarrow P$, and assign $P \mapsto B$. If $B^{\prime} \rightarrow P$ is another special $\mathcal{B}$-precover of $P$ then $B \sim B^{\prime}$ (see Lemma 17), and the claim is clear.

Since general preprojective modules are not classified, Corollary 18 leaves little hope for a complete classification of all Baer modules.

\section{The structure of Mittag-Leffler modules}

Recall that a module $M$ over an arbitrary ring is said to be separable if every finite subset of $M$ is contained in a finitely presented direct summand of $M$.

The torsion-free separable modules over a tame hereditary algebra were studied by Lenzing and Okoh in 25] and 20. We will see that they are precisely the torsion-free (strict) Mittag-Leffler $R$-modules. This will then enable us to give a complete classification of all Mittag-Leffler $R$-modules.

We start by recalling some notions. If $M$ is a right $R$-module, and $\mathcal{Q}$ is a class of left $R$-modules, we say that $M$ is a $\mathcal{Q}$-Mittag-Leffler module if the canonical map

$$
\rho: M \bigotimes_{R} \prod_{i \in I} Q_{i} \rightarrow \prod_{i \in I}\left(M \bigotimes_{R} Q_{i}\right)
$$

is injective for any family $\left\{Q_{i}\right\}_{i \in I}$ of modules in $\mathcal{Q}$. For $\mathcal{Q}=R$-Mod we obtain the notion of a Mittag-Leffler module from [27.

The notion of relative Mittag-Leffler modules appears naturally in connection with cotorsion pairs. For example, if $R$ is a tame hereditary algebra and $\mathcal{C}$ denotes the class of all left $R$-modules without non-zero preinjective summands then, from a general result on cotorsion pairs [5, 9.5], we get the following result

Proposition 19. Let $R$ be a tame hereditary artin algebra. Every Baer right $R$ module is $\mathcal{C}$-Mittag-Leffler. Moreover, a countably generated torsion-free module is Baer if and only if it is $\mathcal{C}$-Mittag-Leffler. 
The next Lemma shows that over an Artin algebra the notions of separable module, Mittag-Leffler module and strict Mittag-Leffler module coincide.

Lemma 20. Let $R$ be a ring. For a given right $R$-module $M$, consider the following statements:

(i) $M$ is separable.

(ii) $M$ is strict Mittag-Leffler.

(iii) $M$ is Mittag-Leffler.

Then $(i)$ implies $($ ii), and (ii) implies (iii). If all finitely presented right $R$-modules are pure injective, then the three statements are equivalent.

Proof. In order to show $(i) \Rightarrow(i i)$ let $\pi: X \rightarrow M$ be a pure epimorphism. If $F$ is a finite subset of $M$ then, by hypothesis, $F$ is contained is a finitely presented direct summand $N$ of $M$. Denote by $\varepsilon: N \rightarrow M$ the inclusion and by $f: M \rightarrow N$ any map such that $f \varepsilon=\mathrm{Id}$. As $N$ is pure projective, there exists $g: N \rightarrow X$ such that $\pi g=\varepsilon$. Set $\varphi=g f$. Then $\pi \varphi(x)=\pi(g f) \varepsilon(x)=\varepsilon(x)=x$ for any $x \in F$. This show that $\pi$ is a locally split epimorphism. Therefore, $M$ is strict Mittag-Leffler.

The implication $(i i) \Rightarrow(i i i)$ is due to Raynaud and Gruson [27]. The remaining part of the statement is due to Zimmermann [35, 2.11].

We now aim at a classification of all Mittag-Leffler modules over a tame hereditary algebra $R$. This is obtained in several steps. We start with a classification of the torsion-free Mittag-Leffler modules. A partial result in this direction was already achieved in [25, 3.1].

Proposition 21. Let $R$ be a tame hereditary artin algebra. The following statements are equivalent for a module $M$.

(1) $M$ is torsion-free (strict) Mittag-Leffler.

(2) $M$ is a locally split epimorphic image of a direct sum of indecomposable preprojective modules.

(3) $M$ is an s-pure submodule of a direct product of indecomposable preprojective modules.

Moreover, the torsion-free (strict) Mittag-Leffler modules form a preenveloping class which is closed under products and pure submodules.

Proof. Let $P$ be the direct sum of the modules in $\mathbf{p}$. It is well known that $P$ is noetherian over its endomorphism ring. This follows from the fact that its dual module is $\Sigma$-pure-injective [19, 4.6] by using [34, Proposition 3 and Observation 8]. By [1, 2.3], a torsion-free module is (strict) Mittag-Leffler if and only if it belongs to the class $\mathcal{G}(P)$ of all locally split epimorphic images of modules in $\operatorname{Add} P$. Moreover, it follows from [1,3.4] that $\mathcal{G}(P)$ is preenveloping and coincides with the class of all s-pure submodules of direct products of finitely generated preprojective modules. The stated closure properties follow from condition (3) and [27, 2.3.3] or [1, 2.3].

Example 22. (1) The modules in Proposition 21 are preprojective, but not necessarily Baer, see Example 16. Also, there are preprojectives that are not separable, see [25, Introduction]. A characterization of pure-projective preprojectives can be found in [25, 2.4].

(2) The module $L$ is an example of a Baer module that is not Mittag-Leffler, because $L$ is countably generated and not pure-projective, see [27, 2.2.2].

Recall that every right $R$-module is a direct sum $X \oplus Y$ of a reduced module $X$ and a divisible module $Y$, see 29 . Next, we investigate the torsion reduced Mittag-Leffler modules. 
Proposition 23. Let $R$ be a tame hereditary artin algebra. The following statements are equivalent for a module $M$.

(1) $M$ is a torsion reduced (strict) Mittag-Leffler module.

(2) $M$ is a locally split epimorphic image of a direct sum of indecomposable finitely generated regular modules.

Proof. Recall that every (strict) Mittag-Leffler module is separable by Lemma 20. $(1) \Rightarrow(2)$ : For every $x \in M$ there is a finitely generated direct summand $M_{x}$ of $M$ containing $x$. Then it is easy to see that the map $\bigoplus_{0 \neq x \in M} M_{x} \rightarrow M$ given by the canonical inclusions $M_{x} \rightarrow M$ is a locally split epimorphism. Moreover, the finitely generated modules $M_{x}$ are again torsion reduced, hence direct sums of modules from $\mathbf{t}$.

$(2) \Rightarrow(1)$ : Of course, the class of all torsion modules is closed under direct sums and epimorphic images. Moreover, using that a module is divisible if and only if it belongs to ${ }^{o} \mathbf{t}$, we easily verify that locally split epimorphic images of modules in Addt are reduced. Hence $M$ is torsion reduced, and it is (strict) Mittag-Leffler by [1, 2.3].

Let us give a further characterization of the torsion-free, and torsion reduced modules, respectively, that are Mittag-Leffler. To this aim, we need some preliminaries. Recall that an inverse system of sets $\left(H_{\alpha}, h_{\alpha \gamma}\right)_{\alpha \gamma \in I}$ is said to satisfy the Mittag-Leffler condition if for any $\alpha \in I$ there exists $\beta \geq \alpha$ such that $h_{\alpha \gamma}\left(H_{\gamma}\right)=h_{\alpha \beta}\left(H_{\beta}\right)$ for any $\gamma \geq \beta$.

It is shown in [27] that a right $R$-module $M$ is Mittag-Leffler if and only if there exists a direct system of finitely presented modules $\left(F_{\alpha}, u_{\beta \alpha}\right)_{\beta \alpha \in I}$ with $M=$ $\lim _{\longrightarrow} F_{\alpha}$ such that the inverse system $\left(\operatorname{Hom}_{R}\left(F_{\alpha}, B\right), \operatorname{Hom}_{R}\left(u_{\beta \alpha}, B\right)\right)_{\beta \alpha \in I}$ satisfies

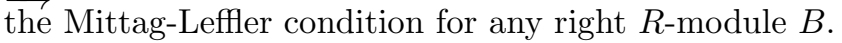

In the next Proposition we present a refinement of this result from [5]. It will allow us to establish a situation in which we can restrict to special choices of $B$ for proving that a module is Mittag-Leffler.

Proposition 24. 5, 6.2] Let $R$ be a ring. Let $\mathcal{B}$ be a class of right $R$-modules closed under direct sums, and let $\mathcal{Q}$ be a class of left $R$-modules. Assume there exists a direct system of finitely presented modules $\left(F_{\alpha}, u_{\beta \alpha}\right)_{\beta \alpha \in I}$ with $M=\lim F_{\alpha}$ such that the inverse system $\left(\operatorname{Hom}_{R}\left(F_{\alpha}, B\right), \operatorname{Hom}_{R}\left(u_{\beta \alpha}, B\right)\right)_{\beta \alpha \in I}$ satisfies the MittagLeffler condition for any right $R$-module $B \in \mathcal{B}$.

If for every $Q \in \mathcal{Q}$ and every $\alpha \in I$ there exists a map $f_{\alpha}: F_{\alpha} \rightarrow B_{\alpha}$ such that $B_{\alpha} \in \mathcal{B}$ and $f_{\alpha} \otimes_{R} Q$ is a monomorphism, then $M$ is a $\mathcal{Q}$-Mittag-Leffler module.

Proposition 25. Let $R$ be a ring, and let $\mathcal{S}$ be a class of finitely presented modules. Let $B$ be a module such that $\mathcal{S} \subseteq \operatorname{Add}(B)$. If $M$ is a module in $\lim \mathcal{S}$, then the following statements are equivalent:

(1) $M$ is Mittag-Leffler.

(2) There exists a direct system of modules $\left(F_{\alpha}, u_{\beta \alpha}\right)_{\beta \alpha \in I}$ in $\mathcal{S}$ with $M=$ $\lim _{\mathrm{F}} F_{\alpha}$ such that the inverse system $\left(\operatorname{Hom}_{R}\left(F_{\alpha}, B\right), \operatorname{Hom}_{R}\left(u_{\beta \alpha}, B\right)\right)_{\beta \alpha \in I}$ $\overrightarrow{\text { satisfies the Mittag-Leffler condition. }}$

(3) For any direct system of finitely presented modules $\left(F_{\alpha}, u_{\beta \alpha}\right)_{\beta \alpha \in I}$ with $M=$ $\lim _{\alpha} F_{\alpha}$, the inverse system $\left(\operatorname{Hom}_{R}\left(F_{\alpha}, B\right), \operatorname{Hom}_{R}\left(u_{\beta \alpha}, B\right)\right)_{\beta \alpha \in I}$ satisfies the $\overrightarrow{\text { Mittag-Leffler condition. }}$

Proof. By [27, 2.1.5] and [5, 4.4] we only have to prove that (3) implies (1).

Fix a direct system $\left(F_{\alpha}, u_{\beta \alpha}\right)_{\beta \alpha \in I}$ such that $F_{\alpha} \in \mathcal{S}$, for any $\alpha \in I$, and satisfying that $M=\lim _{\alpha} F_{\alpha}$. By [5, 3.9], for any $B^{\prime} \in \operatorname{Add}(B)$ the inverse system $\left(\operatorname{Hom}_{R}\left(F_{\alpha}, B^{\prime}\right), \operatorname{Hom}_{R}\left(u_{\beta \alpha}, B^{\prime}\right)\right)_{\beta \alpha \in I}$ satisfies the Mittag-Leffler condition. Since 
$F_{\alpha} \in \operatorname{Add}(B)$, we can apply Proposition 24 , taking $f_{\alpha}=\operatorname{Id}: F_{\alpha} \rightarrow F_{\alpha}$ and $\mathcal{Q}=R$ Mod, to deduce that $M$ is a Mittag-Leffler module.

Now we come back to tame hereditary algebras.

Corollary 26. Let $R$ be a tame hereditary artin algebra. Let $P$ be the direct sum of the modules in $\mathbf{p}$, and let $T$ be the direct sum of the modules in $\mathbf{t}$.

(1) A torsion-free module $M$ is Mittag-Leffler if and only if there exists a direct system of finitely presented modules $\left(F_{\alpha}, u_{\beta \alpha}\right)_{\beta \alpha \in I}$ with $M=\lim _{\alpha} F_{\alpha}$ such that the inverse system $\left(\operatorname{Hom}_{R}\left(F_{\alpha}, P\right), \operatorname{Hom}_{R}\left(u_{\beta \alpha}, P\right)\right)_{\beta \alpha \in I}$ satisfies the Mittag-Leffler condition.

(2) A torsion reduced module $M$ is Mittag-Leffler if and only if there exists a direct system of finitely presented modules $\left(F_{\alpha}, u_{\beta \alpha}\right)_{\beta \alpha \in I}$ with $M=\lim _{\longrightarrow} F_{\alpha}$ such that the inverse system $\left(\operatorname{Hom}_{R}\left(F_{\alpha}, T\right), \operatorname{Hom}_{R}\left(u_{\beta \alpha}, T\right)\right)_{\beta \alpha \in I}$ satisfies the Mittag-Leffler condition.

Proof. The statements are immediate consequences of Proposition 25, because the torsion-free modules are the modules in $\lim _{\longrightarrow}$ add $\mathbf{p}$, and the torsion reduced modules belong to $\underline{\lim }$ add $\mathbf{t}$, see [28, 3.4].

We are now ready to give the announced classification.

Theorem 27. Let $R$ be a tame hereditary artin algebra. The (strict) Mittag-Leffler modules over $R$ are precisely the modules of the form $X \oplus Y$ where $Y$ is a direct sum of indecomposable preinjective modules, and there exists a unique pure-exact sequence

$$
\varepsilon: 0 \rightarrow X^{\prime} \hookrightarrow X \rightarrow X^{\prime \prime} \rightarrow 0
$$

such that $X^{\prime}$ is a locally split epimorphic image of a direct sum of indecomposable finitely generated regular modules, and $X^{\prime \prime}$ is an $(s-)$ pure submodule of a product of indecomposable preprojective modules.

Proof. First of all, we check that every module of the stated form is (strict) MittagLeffler. The module $Y$ is pure-projective, hence strict Mittag-Leffler. The modules $X^{\prime}$ and $X^{\prime \prime}$ are strict Mittag-Leffler by Propositions 21 and 23. Since the class of strict Mittag-Leffler modules is closed under pure extensions [5, 8.12], it follows that $X$ and $X \oplus Y$ are strict Mittag-Leffler.

Conversely, if $M$ is a (strict) Mittag-Leffler module, we write $M=X \oplus Y$ as direct sum of a reduced module $X$ and a divisible module $Y$. Recall that divisible modules are direct sums of indecomposable preinjective modules and of indecomposable infinite dimensional pure-injective modules, see [29]. But no indecomposable infinite dimensional module is separable. Hence $Y$ is a direct sum of indecomposable preinjective modules. As for $X$, we know from [29, 4.1] that there is a unique pure-exact sequence

$$
0 \rightarrow \mathbf{t} X \rightarrow X \stackrel{\nu}{\rightarrow} X / \mathbf{t} X \rightarrow 0
$$

where $\mathbf{t} X=\sum_{f \in \operatorname{Hom}_{R}(Y, X), Y \in \mathbf{t}} \operatorname{Im} f \in$ Gen $\mathbf{t}$ is the trace of $\mathbf{t}$ in $X$, and $X / \mathbf{t} X \in \mathcal{F}$. Since the class of reduced modules is closed under submodules, and the class of Mittag-Leffler modules is closed under pure submodules, we deduce that $X^{\prime}=\mathbf{t} X$ is torsion reduced Mittag-Leffler, hence it has the stated form by Proposition 23. It remains to prove that $X^{\prime \prime}=X / \mathbf{t} X$ is Mittag-Leffler. Then it will have the stated form by Proposition 21. So, we check that $X / \mathbf{t} X$ is separable. Let $0 \neq \bar{z} \in X / \mathbf{t} X$ with $z \in X$. Then $z$ is contained in a finitely generated direct summand of $X$, so we can write $X=A \oplus A^{\prime} \oplus B$ with $A$ finitely generated torsion-free, $A^{\prime}$ finitely generated torsion, and $z=a+a^{\prime} \in A \oplus A^{\prime}$. Since $\operatorname{Hom}_{R}(\mathbf{t} X, A)=0$, we have $\mathbf{t} X \subseteq A^{\prime} \oplus B$, which shows that $X / \mathbf{t} X=\nu(A) \oplus \nu\left(A^{\prime} \oplus B\right)$. Thus $\nu(A)$ is a 
finitely generated direct summand of $X / \mathbf{t} X$ which contains $\bar{z}=\nu(z)=\nu(a)$. This completes the proof.

We recall that over a tame hereditary artin algebra, countably generated MittagLeffler modules are just direct sums of indecomposable finitely generated modules. So if $M$ is a countably generated Mittag-Leffler module then, in the Theorem above, $Y, X^{\prime}$ and $X^{\prime \prime}$ are just the direct sums of the preinjective, regular and preprojective indecomposable direct summands from the direct sum decomposition of $M$.

Mittag-Leffler modules are directed unions of their countably generated MittagLeffler pure submodules [27]. The theorem above explains how the structure of the countably generated submodules is reflected in the whole module.

\section{REFERENCES}

[1] L. Angeleri Hügel: Covers and envelopes via endoproperties of modules, Proc. London Math. Soc. 86 (2003) 649-665.

[2] L. Angeleri Hügel, A key module over pure-semisimple hereditary rings, Journal of Algebra 307 (2007), 361-376.

[3] L. Angeleri Hügel, S. Bazzoni, and D. Herbera, A solution to the Baer splitting problem, to appear in Trans. Amer. Math. Soc.

[4] L. Angeleri Hügel and F. Coelho, Infinitely generated complements to partial tilting modules, Math. Proc. Camb. Phil. Soc. 132 (2002), 89-96.

[5] L. Angeleri Hügel, and D. Herbera, Mittag-Leffler conditions on modules, preprint.

[6] L. Angeleri Hügel, D. Herbera, and J. Trlifaj, Tilting modules and Gorenstein rings, Forum Math. 18 (2006), 211-229.

[7] G. Azumaya, Locally pure-projective modules, Contemp. Math. 124 (1992), 17-22.

[8] R. BAER, The subgroup of the elements of finite order of an abelian group, Ann. of Math. 37 (1936), 766-781.

[9] S. Bazzoni And L. Salce, Strongly flat covers, J. London Math. Soc. 66 (2002), 276-294.

[10] W. Crawley-Boevey, Regular modules for tame hereditary algebras, Proc. London Math. Soc. 62 (1991), 490-508.

[11] W. Crawley-Boevey, Infinite dimensional modules in the representation theory of finite dimensional algebras, CMS Conf. Proc. 23 (1998), 29-54.

[12] P.C. Eklof, L. Fuchs, And S. Shelah, Baer modules over domains, Trans. Amer. Math. Soc. 322 (1990), 547-560.

[13] L. Fuchs And L.SAlce, Modules over Non-Noetherian Domains, AMS, Providence 2001.

[14] W. Geigle, H. Lenzing, Perpendicular categories with applications to representations and sheaves, J. Algebra 144 (1991), no. 2, 273-343.

[15] R. Goebel and J.Trlifaj, Approximations and Endomorphism Algebras of Modules, W. de Gruyter, Berlin 2006.

[16] P. Griffith, A solution to the splitting mixed group problem of Baer, Trans. Amer. Math. Soc. 139 (1969), 261-269.

[17] I. KAPlAnSKY, The splitting of modules over integral domains, Arch. Math. 13 (1962), $341-343$.

[18] O. Kerner And J. TrlifaJ, Tilting classes over wild hereditary algebras, J. Algebra. 290 (2005), 538-556.

[19] H. LenZing, Homological transfer from finitely presented to infinite modules, Lecture Notes in Math. 1006 (1983), 734-761.

[20] H. Lenzing, F. Окон, The separability of direct products of modules over a canonical algebra, Representations of algebras (Ottawa, ON, 1992), 339-352, CMS Conf. Proc. 14, Amer. Math. Soc., Providence, RI, 1993.

[21] F. LukAs, Infinite-dimensional modules over wild hereditary algebras, J. London Math. Soc. 44 (1991), 401-419.

[22] F. Lukas, A class of infinite-rank modules over tame hereditary algebras, J. Algebra 158 (1993), 18-30.

[23] F. Окон, Cotorsion modules over tame finite-dimensional hereditary algebras, in Springer Lecture Notes in Math. 903 (1980), 263-269.

[24] F. Окон, Baer modules, J. Algebra 77 (1982), 402-410.

[25] F. Окон, Separable modules over finite-dimensional algebras, J. Algebra 116 (1988), 400414.

[26] F. Окон, Bouquets of Baer modules, J. Pure Appl. Algebra 93 (1994), 297-310. 
[27] M. Raynaud et L. Gruson, Critères de platitude et de projectivité, Invent. Math. 13 (1971), 1-89.

[28] I. Reiten And C.M. Ringel, Infinite dimensional representations of canonical algebras, Canad. J. Math. 58 (2006), 180-224.

[29] C.M. RingeL, Infinite dimensional representations of finite dimensional hereditary algebras, Symposia Math. 23 (1979), 321-412.

[30] P. Rothmaler, Mittag-Leffler modules and positive atomicity. Habilitationsschrift, Kiel, 1994.

[31] A. Schofield, Representations of Rings over Skew-Fields, Cambridge Univ. Press, Cambridge 1985.

[32] A. Schofield, Universal localization for hereditary rings and quivers, in Springer Lecture Notes in Math. 1197 (1986), 149-164.

[33] H. Strauss, On the perpendicular category of a partial tilting module, J. Algebra 144 (1991), 43-66.

[34] B. Zimmermann-Huisgen, W. Zimmermann, On the sparsity of representations of rings of pure global dimension zero, Trans. Amer. Math. Soc. 320 (1990), 695-711.

[35] W. Zimmermann, On locally pure-injective modules, J. Pure Appl. Algebra 166 (2002), $337-357$.

Dipartimento di Informatica e Comunicazione, Università degli Studi dell'Insubria, Via MaZzini 5, I - 21100 Varese, Italy

E-mail address: lidia.angeleri@uninsubria.it

Departament de Matemàtiques, Universitat Autònoma de Barcelona, E-08193 Bellaterra (BARCElona), Spain

E-mail address: dolors@mat.uab.cat

Charles University, Faculty of Mathematics and Physics, Department of Algebra, Sokolovská 83, 18675 Prague 8, Czech Republic

E-mail address: trlifaj@karlin.mff.cuni.cz 